DOC.

D101.60/5:

2000-07

AKI Kesearcn Ivote 2000-07

\title{
Overview of ARI Recruiting Research
}

Walter C. Borman, Kristen E. Horgen, and Lisa M. Penney

Personnel Decisions Research Institutes

Selection and Assignment Research Unit

Michael G. Rumsey, Chief

January 2000

U.S. Army Research Institute

for the Behavioral and Social Sciences

Approved for public release; distribution is unlimited. 